\title{
Études/Inuit/Studies
}

\section{Notes sur l'inuktitut parlé à Iqaluit (Nunavut)}

\section{Louis-Jacques Dorais}

Volume 26, numéro 1, 2002

Perspectives inuit et qallunaat : points de vue en interaction Inuit and Qallunaaq perspectives: Interacting points of view

URI : https://id.erudit.org/iderudit/009280ar

DOI : https://doi.org/10.7202/009280ar

Aller au sommaire du numéro

\section{Éditeur(s)}

Association Inuksiutiit Katimajiit Inc.

ISSN

0701-1008 (imprimé)

1708-5268 (numérique)

Découvrir la revue

Citer cette note

Dorais, L.-J. (2002). Notes sur l'inuktitut parlé à Iqaluit (Nunavut).

Études/Inuit/Studies, 26(1), 193-201. https://doi.org/10.7202/009280ar

\section{Résumé de l'article}

À partir de tests linguistiques administrés dans le cadre d'une étude sociolinguistique, cette note de recherche décrit un certain nombre de traits phonologiques et morphologiques de l'inuktitut parlé par un échantillon d'adultes et d'enfants vivant à Iqaluit. La note démontre un degré de variation linguistique relativement élevé, surtout en ce qui concerne la morphologie. 


\section{Notes sur l'inuktitut parlé à Iqaluit (Nunavut)}

\section{Louis-Jacques Dorais*}

Abstract: Notes on Inuktitut as spoken in Iqaluit (Nunavut)

Based on linguistic tests conducted in the context of a sociolinguistic study, this research note discusses some phonological and morphological characteristics of Inuktitut, as it is spoken by a sample of adult and child informants living in Iqaluit. The note concludes on a relatively high degree of linguistic variability, especially as morphology is concerned.

Résumé: Notes sur l'inuktitut parlé à Iqaluit (Nunavut)

À partir de tests linguistiques administrés dans le cadre d'une étude sociolinguistique, cette note de recherche décrit un certain nombre de traits phonologiques et morphologiques de l'inuktitut parlé par un échantillon d'adultes et d'enfants vivant à Iqaluit. La note démontre un degré de variation linguistique relativement élevé, surtout en ce qui concerne la morphologie.

\section{Introduction}

Contrairement à ce qui pouvait être le cas il y a une trentaine d'années, la distribution et les caractéristiques principales des langues eskaléoutes et des dialectes inuit sont maintenant bien connues. Des travaux comme ceux de Krauss (1980), Fortescue (1983), Dorais (1990, 1992, 1996), Fortescue et al. (1994) ou, plus récemment, Tersis et Therrien (2000), ont brossé un tableau complet et précis des subdivisions linguales et dialectales du continuum yupik / inuit.

Pour ce qui est de la langue des Inuit, nous savons maintenant qu'elle se subdivise en quatre grands ensembles dialectaux (Alaska, Canada occidental, Canada oriental, Groenland) comprenant chacun de trois à cinq (ou six, selon les classifications) dialectes. L'un de ces ensembles, l'inuktitut de l'Arctique oriental canadien, peut être subdivisé de la façon suivante (Dorais 1996: 52):

Département d'anthropologie, Université Laval, Québec (Québec), G1K 7P4. 
kivalliq (centre-ouest de la baie d'Hudson)

aivilik (nord-ouest de la baie d'Hudson)

nord de Baffin (péninsule de Melville et nord de la Terre de Baffin)

sud de Baffin (sud de la Terre de Baffin)

Nunavik (Québec arctique)

Labrador (côte du Labrador septentrional)

Des travaux plus récents (Dorais 2000: 37; mais voir aussi Fortescue 1983) signalent que certains parlers kivalliq (ceux d'Arviat, au sud du territoire) pourraient appartenir à l'ensemble dialectal de l'Arctique occidental canadien. Des spécialistes de l'inuktitut (Mick Mallon, communication personnelle) considèrent aussi que la langue parlée au sud de la Terre de Baffin comprend en fait deux dialectes: celui de l'est de Baffin (Clyde River, Broughton Island et Pangnirtung) et celui du sud (Kimmirut, Cape Dorset), Iqaluit constituant un carrefour dialectal. C'est cette subdivision qu'a adoptée le dictionnaire électronique Asuilaak, disponible sur Internet (www.livingdictionary.com).

Quoi qu'il en soit, les frontières dialectales sont aujourd'hui beaucoup moins nettes qu'elles ne l'étaient autrefois. En effet, le développement des moyens de transport et des communications dans l'Arctique a facilité les migrations interrégionales et les contacts téléphoniques et radiophoniques, mettant ainsi en relation des gens parlant des dialectes très différents. Dans des centres administratifs tels qu'Inuvik, Cambridge Bay, Rankin Inlet, Iqaluit ou Kuujjuaq, ainsi que dans plusieurs villages plus petits, on trouve maintenant des locuteurs dont les parlers inuit sont d'origines très diverses.

On ne sait pas encore beaucoup de choses sur la situation linguistique réelle de ces communautés pluridialectales, les descriptions existantes de l'inuktitut mettant plutôt l'accent sur la spécificité de tel ou tel parler. Y a-t-il formation d'un dialecte commun, assimilation au dialecte dominant de la région, ou préservation des parlers d'origine des locuteurs? Dans cette note de recherche, je décrirai certaines caractéristiques phonologiques et morphologiques de l'inuktitut parlé par des adultes et des enfants vivant à Iqaluit, la capitale du Nunavut et, avec quelque 5000 habitants, la plus grosse agglomération de l'Arctique canadien. Les données ont été recueillies lors de tests linguistiques administrés dans le cadre du projet de recherche Discourse Practices in the Baffin Region, subventionné par le Conseil de recherches en sciences humaines du Canada et dirigé conjointement par l'auteur et par Susan Sammons du Collège Nunavut de l'Arctique ( $c f$. Dorais et Sammons 2000).

\section{Traits phonologiques}

Le dialecte (ou sous-dialecte) du sud de la Terre de Baffin, qui est celui de la population originaire de la région d'Iqaluit, se caractérise par un certain nombre de traits phonologiques dont certains lui sont propres (Dorais 1975). Le plus connu de ces traits est sans doute le remplacement du phonème/L/ (une latérale fricative sourde, transcrite tou \& en orthographe standard), qu'on retrouve dans le dialecte du nord de la Terre de Baffin ainsi que dans la plupart des parlers inuit du centre et de l'ouest de 
l'Arctique (de même qu'au Labrador et, avec une distribution un peu différente, au Groenland de l'ouest), par le phonème / $t /$. Dans les parlers du sud de Baffin, le «majeur (doigt)» se dira par exemple qitiqtiq, plutôt que qitiq\&iq, comme dans la plupart des autres dialectes. Notons que dans les parlers de Cape Dorset et du Nunavik, /L/ est remplacé par/s/ (qitiqsiq).

Une autre caractéristique de l'inuktitut du sud de Baffin, partagée, celle-ci, avec les dialectes du Nunavik, du Labrador et du Groenland, c'est la gémination des groupes de deux consonnes dont la première était originellement une vélaire. C'est ainsi que le mot ukpigjuaq («harfang des neiges») deviendra uppijjuaq, par gémination des groupes consonantiques $k p$ et $g j$, et iglu («maison») deviendra illu. Notons que ce phénomène est relativement récent. En 1973-74 encore, mes informateurs et informatrices d'Iqaluit et de Pangnirtung, alors dans la quarantaine ou la cinquantaine, avaient tendance à prononcer les consonnes initiales vélaires, la gémination s'entendant surtout dans le parler rapide ou dans la prononciation relâchée des jeunes (Dorais 1975: 1).

Le test linguistique que nous avons fait passer à Iqaluit comprenait quatre éléments visant à mettre en lumière ces deux traits phonologiques. D'une part, nous demandions aux répondants la façon dont ils prononçaient les mots pour «harfang des neiges» et «maison.» D'autre part, ils devaient dire comment ils appelaient le majeur. De plus, nous leur demandions de prononcer le mot pour «lanière,» qui, dans les dialectes du centre et de l'ouest, contient généralement un groupe consonantique composé à la fois d'une vélaire initiale et du phonème /L/ (ak\&unaaq).

Notons dès l'abord que chez nos 50 informateurs adultes, de même que chez les 103 adolescents et enfants d'âge scolaire que nous avons interrogés, il n'y avait pas de différence de prononciation entre hommes et femmes. La variation était plutôt liée au lieu d'origine et, dans une moindre mesure, à l'âge.

Pour ce qui est de la prononciation du mot pour «harfang des neiges» (nous nous sommes arrêté au premier groupe consonantique que ce terme comprend), les résultats sont les suivants chez les adultes:

$\begin{array}{lcc}\text { Nombre } & \begin{array}{c}\text { Nombre } \\ \text { uppi- }\end{array} & \begin{array}{c}\text { ukpi- } \\ \text { Moins de } 30 \text { ans: }\end{array} \\ \text { 30-50 ans } & 15 & 3 \\ 50 \text { ans et plus } & 16 & 2 \\ & 9 & 5\end{array}$

Les quelques personnes de moins de 50 ans prononçant la consonne vélaire (ukpi-) sont originaires du nord de la Terre de Baffin (2 cas), de l'ouest de la baie d'Hudson (2 cas) et, dans un cas, de Broughton Island. Dans les deux premières régions, les vélaires sont encore prononcées aujourd'hui, ce qui semble signifier que ces informateurs continuent à utiliser leur parler d'origine. Broughton Island, par contre, relève du (sous) dialecte de l'est de Baffin. La prononciation de la vélaire par un jeune de moins de 30 ans originaire de ce village pourrait donc constituer un trait conservateur. $\mathrm{Ce}$ 
conservatisme se retrouve chez les personnes de plus de 50 ans, où une proportion nettement plus élevée d'informateurs $(35,7 \%)$ prononce $u k p i-$, alors qu'une seule d'entre elles est originaire du nord de la Terre de Baffin. Notons que chez les répondants de moins de 50 ans utilisant une géminée, on compte quatre personnes originaires du nord de Baffin et deux de l'ouest de la baie d'Hudson, ce qui suggère une certaine assimilation phonologique de leur part.

Une telle assimilation est confirmée par la prononciation du mot pour «maison:»

$\begin{array}{lcc}\text { Âge } & \begin{array}{c}\text { Nombre } \\ \text { illu- }\end{array} & \begin{array}{c}\text { Nombre } \\ \text { iglu- }\end{array} \\ \text { Moins de } 30 \text { ans: } & 14 & 4 \\ \text { 30-50 ans } & 14 & 4 \\ 50 \text { ans et plus } & 13 & 1\end{array}$

Tous les informateurs de moins de 30 ans originaires du nord de la Terre de Baffin géminent le groupe -gl- encore courant dans leur dialecte d'origine. Par contre, il n'en est pas de même des jeunes immigrants de l'ouest de la baie d'Hudson, ni des informateurs plus âgés originaires de régions autres que le sud de Baffin. Il est remarquable que tous les répondants de plus de 50 ans nés dans le sud de la Terre de Baffin géminent le groupe en question, alors que quatre d'entre eux ne géminent pas le -kp- de ukpik. Il semble donc y avoir un certain flottement dans la prononciation des groupes initialement en vélaire, flottement qui pourrait dénoter une assimilation linguistique en cours (tendant vers la gémination), mais non encore complétée.

Un tel flottement n'existe pratiquement pas en ce qui concerne la prononciation des mots pour «majeur» et «lanière:»

Âge

Moins de 30 ans:

$30-50$ ans

50 ans et plus

\section{Âge}

Moins de 30 ans:

30-50 ans

50 ans et plus

\section{Nombre \\ qitiqti- \\ Nombre \\ qitiq\&i-}

10

12

8

attunaa-

9

12

10

4
4
1

ak\&unaa-

4
4
1

\section{Nombre \\ qitiqsi-}

\section{2 \\ 1 \\ 5}

atsunaa-

4

1

2

Presque tous les informateurs prononcent ces deux mots de la façon qui est courante dans leur dialecte d'origine. Ceux qui utilisent le phonème $/ \mathrm{L} / \mathrm{viennent} d \mathrm{du}$ nord de la Terre de Baffin ou de l'ouest de la baie d'Hudson, alors que ceux qui disent qitiqsi- ou atsunaa- sont nés (ou ont un parent né) à Cape Dorset ou au Nunavik. Les exceptions sont deux jeunes (de moins de 30 ans) originaires du nord de Baffin qui 
disent qitiqti-, comme les gens du sud de la région. L'un d'entre eux, par contre, prononce ak\&unaa- avec une fricative sourde. Deux hommes de plus de 50 ans, pourtant originaires du sud-est de la Terre de Baffin, prononcent qitiqsi-, comme les gens de Cape Dorset et du Nunavik, alors qu'un autre (âgé entre 30 et 50 ans), né à Broughton Island (en région sud-est aussi) dit atsunaa-. Un seul informateur, de plus de 50 ans, utilise la vélaire, en prononçant aktunaa-.

Il semble donc que chez les adultes d'Iqaluit, la survivance du phonème /L/ et de sa variante /s/ soit mieux ancrée que celle des groupes consonantiques à initiale vélaire. Ceux-ci se géminent en effet chez plusieurs informateurs, surtout des jeunes, originaires de régions où on l'utilise encore couramment.

La situation est un peu la même chez les adolescents et enfants d'âge scolaire. Sur les 78 d'entre eux ayant répondu à la question concernant le mot pour «harfang des neiges,» 54 (69\%) prononcent uppi- et 24 (31\%) ukpi-. Parmi ces derniers, on retrouve neuf personnes originaires du nord de la Terre de Baffin, mais dix enfants ont toujours vécu à Iqaluit et cinq viennent d'autres villages de la région sud-est. Il semble donc y avoir un certain flottement là aussi. Il est cependant curieux que des enfants et adolescents utilisent une forme (ukpi-) que j'ai qualifiée de conservatrice et qui n'est plus employée, dans la région du sud de la Terre de Baffin, que par quelques résidents âgés. Il se pourrait que les professeurs d'inuktitut considèrent cette forme comme canonique et que ce soit elle qu'on enseigne aux élèves des écoles d'Iqaluit. On aurait ainsi l'explication de sa survivance chez certains jeunes.

Le même phénomène se répète pour ce qui est du mot pour «maison,» que 71 jeunes sur $101(70,3 \%)$ prononcent illu, et $30(29,7 \%)$ iglu. Ici encore, la moitié de ceux qui utilisent la vélaire (15/30) proviennent d'Iqaluit ou d'autres régions où on gémine. Treize des 30 utilisateurs du mot iglu prononcent par contre uppi-, faisant ainsi montre d'un certain flottement puisqu'ils utilisent la vélaire dans un cas et non dans l'autre. L'emprunt par l'anglais de la forme comportant la consonne vélaire (igloo), ainsi que l'influence de l'école, pourraient ici aussi expliquer la survivance de la prononciation iglu chez les jeunes.

La situation est plus claire pour ce qui est du phonème /L/. Sur 59 écoliers ayant prononcé le mot pour «majeur» (44 d'entre eux ne le connaissaient pas du tout), 45 (76\%) ont dit qitiqti-, 12 (20\%) qitiq\&i-, et deux (4\%) qitiqsi- / qitissi- (on n'a pas demandé aux élèves de dire «lanière»). À trois exceptions près (enfants dont la famille est originaire du sud ou de l'est de la Terre de Baffin), les jeunes qui prononcent qitiq\&li- ou qui utilisent/s/ viennent de régions où ces prononciations sont encore courantes. Il semble donc que chez les jeunes comme chez les adultes d'Iqaluit, l'usage du phonème /L/ soit sujet à moins de flottement que la gémination des groupes à initiale vélaire. 


\section{Traits morphologiques}

Parmi les traits morphologiques qui caractérisent le parler du sud de Baffin, nous en avons choisi deux qui peuvent fournir des indications sur la variation linguistique à Iqaluit. Le premier concerne l'expression des cas obliques du possessif, à la première et à la seconde personne du singulier. Dans les dialectes du centre et de l'ouest de l'Arctique, la distinction entre ces deux personnes repose sur une opposition entre les groupes consonantiques -mn- et -ngn-, comme dans iglumnut («vers ma maison») et iglungnut («vers ta maison»). Dans le dialecte du nord de la Terre de Baffin, où le groupe -mn- s'est géminé, il subsiste cependant une distinction entre les formes -nnut («vers mon, ma») et -ngnut (ou -rnut après un radical se terminant par -q; «vers ton, ta»). Au sud de la Terre de Baffin par contre, où les locuteurs géminent généralement les groupes à initiale vélaire (comme -ngn-), il devient impossible de distinguer phonétiquement les deux personnes. Ce phénomène est connu depuis longtemps $(c f$. Schneider 1972: 71, par exemple).

Nous avons demandé à nos informateurs adultes de donner l'équivalent inuktitut des phrases suivantes:

«Vers mon bateau» (ou «dans ma poche»)

umiannut ou ikpiarjunni dans le dialecte du nord de Baffin

«Vers ton bateau» (ou «dans ta poche»)

umiarnut / umiangnut ou ikpiarjungni dans le dialecte du nord de Baffin

Les résultats montrent une variation extrême qui, de prime abord, a peu à voir avec le sexe, l'âge ou le dialecte d'origine:

«Vers mon bateau» («dans ma poche»)

umiannut («vers mon bateau»): 14 cas (ikpiarjunni ou ippiarjunni: 6 cas)

uvanga umianganut («vers son bateau à moi»): 8 cas (uvanga ippiarjungani: 1 cas)

umiarma mitsaanut («vers l'entourage de mon bateau»): 4 cas

umiaranut («vers mon propre bateau»): 2 cas

umiammut (forme «incorrecte» de «vers mon bateau»): 2 cas

umia(r)mut («vers le bateau»): 2 cas

umiarnut («vers ton bateau»): 1 cas

ippiarjunni uvanga («dans ma poche à moi»): 1 cas

«Vers ton bateau» («dans ta poche»)

ivvit umianganut («vers son bateau à toi»): 12 cas (ivvit ippiarjungani: 4 cas)

umiannut («vers mon / ton bateau»): 7 cas

umiarnut («vers ton bateau»): 4 cas (ikpiarjungni ou ippiarjungni: 3 cas)

umiaqpit / umiavit mitsaanut («vers l'entourage de ton bateau»): 4 cas

umiamut («vers le bateau»): 1 cas

umiarminut («vers son propre bateau»): 1 cas

ippiarjunniivvit («dans ta poche à toi»): 1 cas 
Malgré la variation constatée, certaines constantes se dégagent de l'expression des première et deuxième personnes du possessif. Pour une quinzaine d'informateurs, la distinction entre personnes oppose une forme en -nn- (première personne) et une construction (ivvit -ngan-; «son, à toi») permettant de contourner la neutralisation morphologique qu'entraîne la gémination. Notons que neuf répondants utilisent aussi une telle construction (uvanga-ngan-) à la première personne.

Tous les informateurs de 50 ans et plus utilisent la forme originelle (-nnut, -nni) à la première personne. Par contre, à la deuxième personne, trois d'entre eux ont recours à la construction ivvit -ngani, et le seul à employer la forme originelle umiarnut vient du nord de la Terre de Baffin. En fait, à une exception près (un homme d'âge moyen originaire du sud-est de la Terre de Baffin), les sept personnes utilisant la forme originelle de la deuxième personne (umiarnut, ikpiarjungni) ont émigré du nord de Baffin ou de l'ouest de la baie d'Hudson. Les gens du sud de la Terre de Baffin semblent donc avoir apporté plusieurs solutions différentes au dilemme morphologique posé par la gémination de la vélaire.

Une telle variation existe aussi en ce qui concerne l'expression des cas modalis (ou accusatif) et ablatif de la déclinaison des noms. Les grammaires de l'inuktitut enseignent que la plupart des dialectes distinguent entre une forme -mik, qui exprime le fait que le nom est complément d'objet direct, et une forme -mit, qui exprime la provenance, comme dans iglumik takuvunga («je vois une maison») et iglumit qaivunga («je viens de la maison»). Dans le parler du sud de Baffin cependant, plusieurs locuteurs ne font plus de différence formelle entre le modalis et l'ablatif, comme c'est aussi le cas en langue yup'ik du sud-ouest de l'Alaska. Chez nos informateurs adultes d'Iqaluit, le modalis et l'ablatif s'expriment de la façon suivante:

Modalis / ablatif:

-mik / -mit: 14 cas

$-m i /-m i$ : 12 cas

$-m i k /-m i k: 8$ cas

-mit / -mit: 5 cas

$-m i k$ / -mi: 2 cas

$-m i$ / -mit: 6 cas

$-m i$ / -mik: 1 cas

-mit / -mi: 1 cas

Huit des 14 informateurs qui distinguent toujours entre les formes -mik et -mit sont originaires de l'extérieur de la région du sud de la Terre de Baffin. La moitié des répondants (25 / 49), y compris plusieurs personnes qui ne viennent pas de la région, ne font plus de distinction formelle entre le modalis et l'ablatif. On compte parmi eux sept personnes de plus de 50 ans, soit la moitié des 14 informateurs appartenant à ce groupe d'âge. L'expression de ces deux fonctions syntaxiques semble donc en voie de transformation chez les adultes d'Iqaluit. 
Elle l'est aussi chez les enfants où, sur 14 écoliers ayant répondu à une question sur l'expression de l'ablatif, la moitié (7 / 14) utilise la forme originelle -mit, cinq la forme -mik et deux la forme -mi.

À partir des exemples présentés ici, on peut donc constater une variation morphologique et, à un moindre degré, phonologique assez marquée chez les adultes et les jeunes d'Iqaluit, variation qui montre, entre autres, l'importance des phénomènes de gémination. Certaines personnes originaires - ou dont les parents sont originaires - de régions autres que le sud de la Terre de Baffin continuent à utiliser des formes caractéristiques de ces régions; d'autres non. La seule conclusion qu'on puisse avancer pour l'instant c'est qu'il y a là matière à plus ample recherche. On en sait encore peu sur les formes d'inuktitut aujourd'hui parlées à Iqaluit, et la langue en usage dans cette communauté, comme celle qui est parlée dans d'autres carrefours de l'Arctique contemporain, devrait faire l'objet d'études plus exhaustives.

\section{Références}

DORAIS, Louis-Jacques

1975 Iqalungmiut uqausingit / Southeast Baffin Inuit Language / Le parler inuit de Baffin sud-est, manuscrit dactylographié, Québec, Association Inuksiutiit katimajiit.

1990 Inuit uqausiqatigiit. Inuit Languages and Dialects, Iqaluit, Nunavut Arctic College.

1992 La situation linguistique dans l'Arctique, Études/Inuit/Studies, 16(1-2): 237-255.

1996 La parole inuit. Langue, culture et société dans l'Arctique nord-américain, Paris, Éditions Peeters.

2000 Présentation géolinguistique et sociolinguistique de la famille eskaléoute, in $\mathrm{N}$. Tersis et M. Therrien (dir.), Les langues eskaléoutes, Paris, CNRS Éditions: $31-50$.

DORAIS, Louis-Jacques et Susan SAMMONS

2000 Discourse and Identity in the Baffin Region, Arctic Anthropology, 37(2): 92-110.

FORTESCUE, Michael

1983 A comparative manual of affixes for the Inuit dialects of Greenland, Canada and Alaska, Copenhague, Meddelelser om Grønland (Man and Society 4).

FORTESCUE, Michael, Steven JACOBSON et Lawrence D. KAPLAN

1994 Comparative Eskimo Dictionary. With Aleut Cognates, Fairbanks, University of Alaska, Alaska Native Language Center. 
KRAUSS, Michael E.

1980 Alaska Native Languages: Past, Present, and Future, Fairbanks, University of Alaska, Alaska Native Language Center (Research Paper no 4).

SCHNEIDER, Lucien

1972 Inuktituorutît. Grammaire purement esquimaude, Québec, Direction générale du Nouveau-Québec.

TERSIS, Nicole et Michèle THERRIEN (dir.)

2000 Les langues eskaléoutes. Sibérie, Alaska, Canada, Groënland, Paris, CNRS Éditions. 